\title{
To Quantize or Not to Quantize: Fact and Folklore in Quantum Gravity*
}

\author{
Christian Wüthrich \\ University of Pittsburgh
}

30 September 2004

\begin{abstract}
Does the need to find a quantum theory of gravity imply that the gravitational field must be quantized? Physicists working in quantum gravity routinely assume an affirmative answer, often without being aware of the metaphysical commitments that tend to underlie this assumption. The ambition of this article is to probe these commitments and to analyze some recently adduced arguments pertinent to the issue of quantization. While there exist good reasons to quantize gravity, as this analysis will show, alternative approaches to gravity challenge the received wisdom. These renegade approaches do not regard gravity as a fundamental force, but rather as effective, i.e. as merely supervening on fundamental physics. I will urge that these alternative accounts at least prove the tenability of an opposition to quantization.
\end{abstract}

\section{Introduction}

All major approaches to quantum gravity endorse, implicitly or explicitly, the view that gravity must be "quantized" in order to be amenable to a description at the fundamental level. To be sure, different approaches propose to execute this task in radically different manners. But they agree

\footnotetext{
*I wish to thank John Earman, Florian Girelli, John Moffat, Carlo Rovelli and Danny Terno for discussions. This work is supported by the Swiss National Science Foundation (grant PBSK1-102693).
} 
in that without a quantization of gravity no consistent quantum theory of gravity will be forthcoming. What such a quantization involves will vary with the approach taken. But rather than analyzing what is meant by "quantization" - be it some definite procedure for converting a classical field into a quantum field, or the discreteness of spacetime $a b$ initio-I shall focus on the intriguing foundational issues that arise in the context of motivating and justifying such a quantization of gravity.

Callender and Huggett (2001a, 2001b) have sensibly suggested to distinguish between the two separate issues of motivating the quest for a quantum theory of gravity on the one hand and motivating the quantization of the gravitational field on the other. The first problem can be expressed by asking "why do we need a quantum theory of gravity at all?", and the second, assuming that the first one was answered in the affirmative, by inquiring "why do we have to quantize gravity for the purpose of finding a quantum theory of gravity?". It may seem that these two questions can hardly be kept separate since quantum field theory requires that all matter fields be quantized and general relativity teaches that those matter fields are the sources for the gravitational field. Whether or not the two questions collapse into one depends on whether it will turn out to be possible that quantum matter fields coexist with the classical, i.e. non-quantized, gravitational field. Since at least some approaches to gravity, the so-called semi-classical theories, insist that such coexistence is indeed possible, distinguishing the two questions will help to map the debate.

So why do we need a quantum theory of gravity? A strong, but nevertheless often nebulous, desire to present a unified theoretical framework at the level of fundamental physics populates the folklore of physicists and often fuels the search for a quantum theory of gravity. Arguments to this effect, relying - if made explicit at all —on metaphysical considerations, typically elicit some principles of unity of nature or of scientific method. Although general relativity and quantum theory may be so disparate as to disallow the formal deduction of contradictions, they are generally taken to be incommensurable (families of) theories. A quantum theory of gravity is expected to remedy this theoretical schism and to bolster attempts at finding the Holy Grail of physics, a unified framework of all interactions. The argument from unification - unification for the sake of unification - does not, however, sway the sceptic. The "disunitist" would certainly be free to respond that at the very least, it may just as well be the case that the conceptual disunity of the two theories reflects a disunity in nature. Despite its rare explicit articulation and its questionable metaphysical strength, the unificatory impetus provides an extremely important motivation for attempts at 
quantizing gravity. ${ }^{1}$

But it suffices to imply the resources of physics itself to find perfectly valid objectives to do quantum gravity. Extant theoretical physics strongly motivates the search for a quantum theory of gravity by itself and thus dispenses with the need to invoke metaphysical unitism. General relativity conceives of gravity as a dynamical field capturing the gravitational dance of classical matter, while quantum theory maintains that all dynamical fields must be quantized, i.e. that all matter is quantum. Although this tension may be mostly dormant at the energies that are currently experimentally accessible, there are situations where the interaction between the matter fields qua quantum fields and the gravitational field becomes pertinent. For example, physically realistic cosmological models generically predict an initial singularity. At this singularity, classical physics breaks down and it is assumed that a quantum theory of gravity, i.e. a theory combining general relativity with quantum physics, will be necessary to probe the physics of the early universe.

Given the lack of empirical indications that a quantum theory of gravity is necessary to correctly describe the physics in these exotic regimes, one might be inclined to dismiss the endeavor as mathematical recreation. So far, the only empirical input is extremely indirect at best, involving an uncomfortably large number of substantial auxiliary hypotheses to relate the data to Planck scale physics. But despite the fact that the energies required to probe the Planck scale directly continue to be out of reach, a growing number of research groups studies the phenomenology of quantum gravity. In particular, data pertinent to the detection of the breaking of Lorentz symmetry, which some claim is implied by some approaches to quantum gravity, is currently under scrutiny (see Jacobson et al. 2003). It certainly seems that impending empirical input might very soon substantiate the need for a quantum theory of gravity.

Notwithstanding some potential reservations regarding the empirical accessibility, the need for a theory merging quantum theory and general relativity is universally acknowledged. Opinions part, however, when the floor is opened to discussions as to how the problem of constructing such a theory must be approached. This essay contends that there are powerful arguments which strongly suggest that gravity should indeed be quantized. Terno's result in particular, to be discussed in Section 2, provides such an argument. The same section also deals with what is probably the most common litany

\footnotetext{
${ }^{1}$ Mattingly (1999) discusses some stipulated or implied unity principles and relates it to the unity of science debate in philosophy of science.
} 
defending the necessity of a discrete spacetime structure. I analyze one of its best expressions - the one due to Doplicher and collaborators - and conclude that it begs the questions. Common to both Terno's and Doplicher's arguments is their attempt of solely drawing on resources from physical theories and their mathematical apparatus rather than relying on metaphysical or aesthetic principles. These arguments favorable to quantization will be contrasted in Section 3 with two alternative approaches to quantum gravity which do not involve quantization and by their mere existence establish the contingency of the quantization of gravity. My conclusions follow in Section 4.

\section{Why quantize gravity?}

Assuming then that the need for a quantum theory of gravity has been established, does gravity necessarily have to be quantized in such a theory? I concur with Callender and Huggett (2001a, 2001b) and with Mattingly (1999) that this question must be answered in the negative. The mere existence of approaches to quantum gravity which do not involve the quantization of gravity implies that quantization is a contingent matter. Any semi-classical theory of quantum gravity, which supplies the wedge that Callender and Huggett drive between the two disparate questions of whether we need a quantum theory of gravity and of whether such a need implies the quantization of gravity, constitutes such an approach. In its simplest form, the theory stipulates the coupling of the classical gravitational field to the quantum fields of matter. The Einstein equations are modified by exchanging the classical energy-momentum density $T_{\mu \nu}$ with its expectation value given the quantum state of matter $\psi$, i.e. we have the semi-classical Einstein equations

$$
G_{\mu \nu}=8 \pi\left\langle\psi\left|T_{\mu \nu}\right| \psi\right\rangle
$$

where the $G_{\mu \nu}$ characterize the spacetime geometry. Thus, quantum matter can be coupled to the classical spacetime geometry via the "classical" quantity $\left\langle\psi\left|T_{\mu \nu}\right| \psi\right\rangle$. Semi-classical quantum theories of gravity may or may not include quantum backreactions, i.e. quantum fluctuations induced on the (classical) gravitational field by its coupling to the quantum fields of matter. Those which lack quantum backreactions yield cosmological models which are much too uniform as compared to observations (Boucher and Taschen 1988, 3525). Those which include them, face the problem of consistenly combining two dynamical sectors, following the classical Einstein 
and the quantum Schrödinger evolution respectively. Furthermore, as will be discussed in Section 2.2, they are subject to Terno's no-go result.

There is, overall, little doubt that in the final quantum theory of gravity it will be quantized, but the question will ultimately have to be settled on empirical grounds. Thus, the main conclusion of Callender and Huggett stands. However, their investigation is too narrow in that it is confined to Eppley and Hannah's classic, but somewhat obsolete argument. Eppley and Hannah (1977) have argued that gravity must be quantized on the basis that the interaction of a classical gravitational field with a quantum field leads to contradictions with trusted physical principles. Callender and Huggett detect some loopholes in the argumentation of Eppley and Hannah and consequently discount it as incomplete. My main reservation against Eppley and Hannah stems from their argument's dependence on the interpretation of quantum mechanics. Even if there were no loopholes at all, I urge, any argument based on one specific, highly controversial interpretation of quantum mechanics - the collapse interpretation in this case - suffers by extension. In toto, of course, I concur with Callender and Huggett in that Eppley and Hannah's case for quantization remains inconclusive.

Here, I propose to undertake an analysis of some more recent, and, I believe, more powerful, arguments that have been brought forth on both sides.

\subsection{Non-commutative spacetime operators}

The most popular argument for the non-commutativity of the spacetime coordinates and for the discreteness of spacetime dates back to Snyder (1947) and has found a famous expression in Doplicher et al. (1995). This discreteness encapsulates the quantum nature of spacetime or, equivalently, of the gravitational field. Doplicher and collaborators have argued that the quantum uncertainty relations of the spacetime coordinates emerge from the combination of Heisenberg's principle with general relativity. The argument, as I interpret it, should contain two main parts: the first shows that continuous spacetime loses its operational meaning at small scales already at the semi-classical level; the second would extend the argument to full quantum gravity by defending that this operational limit is due to the truly discrete nature of spacetime at Planck scale.

The first part of the argument encodes a rather common belief that the combination of the uncertainty relations and classical general relativity imposes restrictions on the operational meaning of classical spacetime. The reasoning runs along the following lines. If 
1. the greater the accuracy (or, equivalently, the smaller the uncertainty) in the measurement of spatio-temporal coordinates, the stronger the gravitational field generated by the measurement, and

2. an increasing gravitational field eventually becomes strong enough as to collapse to a black hole, creating a closed trapped surface, and thus prevents any signal from leaving the region at stake, and

3. an operational meaning can only be attached to a spacetime localization in case signals can leave the region measured,

then a localization measurement is operationally restricted to a certain maximum accuracy, or minimum uncertainty. The third premise is merely a definition of operational meaning and does not seem to be either problematic or substantial. The first two premises, on the other hand, are those that procure the mileage. The second premise epitomizes how gravity determines the causal structure of spacetime. This is an immediate consequence of classical general relativity and deserves all the credit that general relativity does. The first premise is a rather direct consequence of combining Heisenberg's uncertainty principle with the principles of general relativity. According to the uncertainty principle, measuring a spacetime coordinate with accuracy $1 / E$ generates an uncertainty $E$ in the associated momentum. Thus, an uncontrollable energy of the order $E$ is concentrated in the region to be localized. According to general relativity, the energy-momentum density $T_{\mu \nu}$ associated with this energy deforms the geometry of the spacetime, as encoded in Einstein's unmodified field equations $G_{\mu \nu}=8 \pi T_{\mu \nu}$. Note that the first two premises are implied by basic principles of well-trusted physical theories. While this certainly does not make them unassailable, our reasons for rejecting them would have to be substantial enough to challenge first principles of two of the most successful achievements of twentieth-century physics: quantum mechanics and general relativity.

One may argue, however, that these first principles will naturally be challenged at the level of quantum gravity. The fact that attempts to combine quantum mechanics with general relativity unveil their conceptual incommensurability can be taken to imply that some of their first principles must be violated in quantum gravity. In a sense, then, quantum mechanics and general relativity when combined already contain the seeds of their own destruction. Thus, one cannot expect that all principles that underpin the above premises will still be valid in full quantum gravity. But if not all premises hold, then the argument will of course collapse. Because the source energy associated with $T_{\mu \nu}$ is quantum and the argument as given is 
thus strictly semi-classical, the objection could conclude, it may very well turn out that the discreteness emerges only as an artefact of the manner in which quantum mechanics and general relativity were combined at the semi-classical level. Therefore, the argument as given so far must be complemented by a second part asserting that the operationally discrete spacetime at the semi-classical level results from an underlying discreteness at the fundamental Planck level.

Unfortunately, Doplicher and his collaborators do not provide such an addition. But neglecting to take this second leg seriously amounts to begging the question. It is undoubtedly true that if spacetime is discrete at the Planck level, it is reasonable to expect some signatures of this discreteness to surface at the semi-classical level. But the converse is not true, exactly because some or all of the premises made above may no longer obtain in full quantum gravity. The argument as it stands will hence not make any converts. Despite its appealing reliance on deeply entrenched physical principles, the argument thus falls short of proving that spacetime must be discrete (or, similarly, that gravity must be quantized) from the resources of trusted physical theories alone.

But the question then becomes how any argument drawing solely on accepted physical theories can possibly establish that gravity must be quantized. If a quantum theory of gravity would be part of the established corpus of theories, the proof would be easy. But alas, it is not! The failure of current physics to offer a straightforward and unique path to a quantum theory of gravity strongly suggests that the formulation of such a theory will require new physics. In this case, however, one cannot accept an argument from the resources of current physics alone to the effect that gravity must be quantized.

\subsection{Inconsistency of quantum-classical dynamics}

Despite this limitation of arguments relying on principles of accepted physics, some ways of constructing a quantum theory of gravity without quantization can still be excluded. Building on earlier results, Terno (2004) has brought forth an argument which seems to preclude the possibility of a consistent semi-classical quantum theory of gravity which includes quantum backreactions. Consider a system consisting of two sectors, one governed by quantum field theory (the quantum sector), and a classical sector described by so-called Koopmanian dynamics. Koopmanian dynamics offers a generalized formalism that allows to cast a classical system in the mathematical formalism of a quantum theory, i.e. as a Hamiltonian system living 
in a Hilbert space. The conjugate variables of the classical sector will be represented by commuting multiplication operators, as opposed to the noncommuting operators of the quantum sector. Furthermore, assume that the hybrid dynamics of the combined classical-quantum system is described by a unitary evolution on the joint Hilbert space $\mathcal{H}=\mathcal{H}_{q} \otimes \mathcal{H}_{c}$.

Terno shows that if we include terms with unobservable classical operators in the interaction part of the unitary operator, then the equations of motion of the combined system will no longer exhibit formal equivalence with the purely classical or the purely quantum equations of motion. But such a formal correspondence is required, he argues, in order to obtain the correct classical limit that is expected to hold for the combined system. Therefore, he continues, the interaction part can only contain observable classical operators. If this is the case, he concludes, the quantum sector cannot influence the classical sector, thus precluding quantum backreactions. Even under these relatively weak assumptions it thus turns out to be impossible to define a mixed system with a hybrid dynamics that consistently includes the full interaction between the two sectors. The looming inconsistency that would arise when quantum backreactions are included in the interaction part of the evolution operator is that the system would exhibit an incorrect classical limit.

Although Terno's result also inhabits the semi-classical realm, I maintain that this does not render it vulnerable to the above charges as it did the preceding argument. The reason is simple: it does not compound two theories on whose principles it relies, but only offers a no-go result using a general formalism and invoking the unitarity of the combined evolution as well as the requirement for a quantum theory of gravity to possess the correct classical limit. These principles, it seems, constitute general desiderata for constructing theories rather than axioms of a specific theory that may become redundant as the theory is superseded. A full quantum theory of gravity is thus likely to also be subjected to these requirements. Insofar as Terno's assumptions transcend particular theories, they are immune to the objections aired above.

\section{Why not quantize gravity?}

Terno's result precludes the possibility of formulating a consistent semiclassical quantum theory of gravity including full quantum backreactions. But there exist more promising alternative approaches that offer quantum theories of gravity which do not involve a quantization of the gravitational 
field and, I will argue, escape Terno's no-go theorem. Typically, they understand gravity as an induced rather than a fundamental force. According to this view, gravity is not one of the four fundamental forces; instead, it emerges at a higher level as a result of the fundamental physics. For example, Lorentz (1899-1900) suggested that Newtonian gravity could be a residual net force supervenient on the electromagnetic force. In this section, I expose two newer proposals which, at least to some extent, do not regard gravity as a fundamental force. For them, since gravity is not fundamental, it does not have to be quantized. Thus, they provide quantum theories of gravity while denying the necessity of its quantization.

The reader may wonder why I wish to label these approaches as quantum theories of gravity. Obviously, they do not constitute theories of gravity qua quantum field. But even so, they offer a resolution of the conceptual tension between quantum theory and general relativity and promise to restitute an account of those astrophysical phenomena which led us to quest for a quantum theory of gravity in the first place. Hence, they solve the problem that required a quantum theory of gravity - a combination of quantum theory and general relativity-, and shall thus be termed as such.

\subsection{Sakharov's induced gravity}

Most prominently, perhaps, among these alternative approaches is Sakharov's induced gravity theory. As it has recently been resurrected, it deserves particular attention. ${ }^{2}$ It claims to ingest Lorentz's vision of the possibility of gravity merely being an effective force induced by residual electromagnetic forces. For Sakharov, gravity is thus not a fundamental physical field, but "induced", i.e. emergent from quantum field theory like hydrodynamics emerges from molecular physics. Nota bene, since the interaction part of the action contains both classical and quantum terms, Sakharov's account leads to a type of semi-classical quantum gravity.

The general framework for an induced gravity theory in Sakharov's vein is set up by first assuming a Lorentzian manifold as a background on which to do quantum field theory. This background is a continuous, classical, unquantized spacetime. It is left free "to flap in the breeze", i.e. no assumptions regarding its dynamical evolution are made. In particular, no Einstein equations - modified or not - enter the picture. When we do quantum field theory on this background spacetime, it turns out that the effective action

\footnotetext{
${ }^{2}$ The original paper is Sakharov ([1967/8] 2000). There is a modern recast by Visser (2002). Sakharov's original article had almost 320 citations in high energy physics as of September 2004, of which 24 in 2003 alone.
} 
at the one-loop level automatically contains terms proportional to the cosmological constant and to the Einstein-Hilbert action of general relativity, as well as higher order terms. Thus, it looks as if Einstein gravity will be generated at the one-loop level from the interaction of quantum fields.

It may be instructive to note that gravity was not really created ex nihilo. At the bare minimum, a Lorentzian background manifold was assumed. Furthermore, the geometry of the background manifold acted as an external field for the quantum fields living on the background manifold. Terms encoding the geometry of the classical background thus cohabited in the Lagrangian of the effective action with the terms of the quantum fields. To the extent to which the background geometry was preseupposed, arguably, the gravitational degree of freedom, but not its dynamics, was put into the theory from the start. The next step, the variation of the effective action with respect to its variables then automatically leads to the semi-classical Einstein equations (1). While the fact that the Einstein-Hilbert action is mimicked at the one-loop level is very suggestive, it is unclear whether this framework is sufficient to fully recover gravity. But it is important to note that gravity, although arguably not fully "induced", was not quantized, as the geometry was assumed to be classical. Furthermore, the approach avoids Terno's prohibition of a semi-classical theory of gravity with quantum backreactions. Since no dynamical assumptions were made regarding the classical background (the classical sector), i.e. no classical dynamics imposed on the classical sector, the gravitational action is induced from the mutual interaction of the underlying quantum fields. The classical sector acts as a mere background and does not interact with the quantum sector. Therefore, no hybrid dynamics is necessitated in this approach.

Interestingly, there exist reverse efforts of constructing a fundamental quantum theory of gravity, subsumed under the heading "non-commutative geometry", which assume only gravity to be a fundamental force and derive the standard model of electromagnetic, weak, and strong forces from gravity. However widespread and persuasive the belief in a fundamental theory containing gravity as a fundamental force may be, currently available observations and experiments only license inferences regarding the same semiclassical realm that Sakharov's approach describes. Not unlike the belief in the unity of nature, the belief in a fundamental theory including gravity is exposed by Sakharov as an additional commitment not warranted by the resources of empirical physics alone. 


\subsection{Jacobson's gravitational thermodynamics}

More recently, Jacobson (1995) has offered a perspective that also cautioned against quantizing the Einstein equations. Rather than deriving the four laws of black hole thermodynamics from the classical Einstein equations, as did Bardeen, Carter, and Hawking (1973), Jacobson inverts the derivation by recovering the Einstein equations from the proportionality of entropy and horizon surface area of a black hole together with the fundamental thermodynamical relation $\delta Q=T d S$, connecting heat $Q$, temperature $T$, and entropy $S$. The heat is interpreted as the energy flux across a causal horizon and the temperature as the Unruh temperature relative to an accelerated observer just inside a local Rindler horizon. This heat manifests itself via the gravitational field it generates. Like in conventional thermodynamics, where heat is interpreted as energy flux between unobservable degrees of freedom, the underlying mechanics of the energy flux is irrelevant. Assuming cosmic censorship, Jacobson formulates local gravitational thermodynamics for an observer by means of the boundary of her past (her "causal horizon"), associating this boundary with entropy. The system that radiates heat is identified with the degrees of freedom behind the horizon, separated from the observer's past by a causality barrier and is therefore unobservable.

As Jacobson shows, this interpretation imposes conditions on the curvature of spacetime such that the classical Einstein equations are implied. Therefore, he suggests that the Einstein equations can be more adequately analogized with the wave equation for sound in a medium, rather than interpreted as the dynamical equations for a fundamental field. These equations, he urges, as higher-level equations of state, should then not be quantized as if the gravitational field were fundamental, despite the fact that they may describe what is ultimately a quantum reality.

Like in Sakharov's approach, gravity does not represent a fundamental force. Rather, it emerges as a phenomenon supervenient on the energy flux from causally inaccessible degrees of freedom. Again, Terno's result does not apply. However, Jacobson's claim that gravity should not be quantized in this scheme because it represents a collective, higher-order degree of freedom is simply false. Physicists routinely quantize collective degrees of freedom such as sound (with "phonons" as quanta of sound). Whether or not a degree of freedom must be quantized or not does not depend on whether it is collective or individual, but on altogether different considerations. Hence, quantization cannot necessarily be escaped by Jacobsonian gravity; but it is not forced on it either.

Jacobson's incipient program, unfortunately, has so far not been worked 
out in any detail. It remains to be seen, therefore, whether it will be able to offer a full picture of the workings of gravity. If it will be successful in this undertaking, then the quantizers of gravity may have met a challenge.

\section{Conclusion}

This paper has analyzed four arguments pertinent to the issue of whether gravity must be quantized in a final quantum theory of gravity, two on each side of the debate. The argument exemplified by Doplicher and collaborators, prevalent in the physics community - as far as the community is concerned with the issue at all-, has been shown to be incomplete. It was charged with lacking a part establishing that the operationally discrete spacetime results from an underlying discreteness at the Planck scale. Next, I have accepted Terno's argument as a proof of the impossibility of constructing a consistent semi-classical theory including truly hybrid dynamics. I insisted, however, that it does not rule out accounts where gravity is not a fundamental, but only an effective force.

Sakharov's induced gravity program provides an effective, semi-classical approach to gravity that does not require a quantization of gravity. Similarly, Jacobson's gravitational thermodynamics conceives of gravity as emergent from the energy flux of unobservable degrees of freedom. Again, no quantization was necessary. Although regret must be expressed that these accounts are but nascent attempts to understand gravity and that they are therefore yet lacking in many respects, they establish that it is at least conceivable that the final theory of gravity may not involve quantization.

The question of whether the gravitational field must be quantized in a full quantum theory of gravity thus turns out to be more subtle than is commonly assumed. Once stripped from its endemic folkore, the field affords a wide variety of arguments drawing on the resources of physical theories. None of these arguments can, by itself, claim conclusiveness. But they all promise to address a multitude of foundational issues encountered in the Herculean task of formulating a quantum theory of gravity.

\section{References}

[1] Bardeen, James M., Brandon Carter, and Stephen W. Hawking (1973), "The Four Laws of Black Hole Mechanics", Communications in Mathematical Physics 31: 161-170. 
[2] Boucher, Wayne, and Jennie Taschen (1988), "Semiclassical physics and quantum fluctuations", Physical Review D37: 3522-3532.

[3] Callender, Craig, and Nick Huggett (eds.) (2001a), Physics Meets Philosophy at the Planck Scale, Cambridge: Cambridge University Press.

[4] - (2001b), "Why Quantize Gravity (or Any Other Field for That Matter)?", Philosophy of Science 68 (Proceedings): S382-394.

[5] Doplicher, Sergio, Klaus Fredenhagen, and John E. Roberts (1995), "The Quantum Structure of Spacetime at the Planck Scale and Quantum Fields", Communications in Mathematical Physics 172: 187-220. Also available at arxiv.org/hep-th/0303037.

[6] Eppley, Kenneth, and Eric Hannah (1977), "The Necessity of Quantizing the Gravitational Field", Foundations of Physics 7: 51-68.

[7] Jacobson, Ted (1995), "Thermodynamics of Spacetime: the Einstein Equation of State", Physical Review Letters 75: 1260-1263. Also available at arxiv.org/gr-qc/9504004.

[8] Jacobson, Ted, Stefano Liberati, and David Mattingly (2003), "A Strong Astrophysical Constraint on the Violation of Special Relativity by Quantum Gravity", Nature 424: 1019-1021. Extended version available at arxiv.org/astro-ph/0212190.

[9] Lorentz, Hendrik A. (1899-1900), "Considerations on Gravitation", Koninklijke Akademie van Wetenschappen te Amsterdam. Proceedings 2: 559-574. Reprint. Originally published as "Beschouwingen over de zwaartekracht", Koninklijke Akademie van Wetenschappen te Amsterdam. Verslagen van de Gewone Vergaderingen 8: 603-620.

[10] Mattingly, James (1999), "Is Quantum Gravity Necessary?", Talk delivered at the Fifth International Conference on the History and Foundations of General Relativity, 9 July 1999.

[11] Sakharov, Andrei D. ([1967/8] 2000), "Vacuum Quantum Fluctuations in Curved Space and the Theory of Gravitation", General Relativity and Gravitation 32: 365-367. Reprint. Originally published in Doklady Akademii Nauk SSSR 177(1967): 70-71, and in Soviet Physics Doklady 12(1968): 365-367.

[12] Snyder, Hartland S. (1947), "Quantized Space-time", Physical Review 71: 38-41. 
[13] Terno, Daniel R. (2004), "Inconsistency of quantum-classical dynamics, and what it implies", Foundations of Physics, forthcoming. Also available at arxiv.org/quant-ph/0402092.

[14] Visser, Matt (2002), "Sakharov's Induced Gravity; a Modern Perspective", Modern Physics Letters A17: 977-992. Also available at arxiv.org/gr-qc/0204062. 